\title{
Pregnancy Counseling for Kidney Transplant Recipients
}

\author{
Yuki Yoshikawa $^{1^{*}}$, Junji Uchida ${ }^{2}$, Akihiro Kosoku$^{2}$, Chiharu Akazawa ${ }^{3}$, Nobuhiko Suganuma ${ }^{4}$ \\ ${ }^{1}$ Shitennoji University, Osaka, Japan; ${ }^{2}$ Department of Urology, Osaka City University Graduate School of Medicine, Osaka, Japan; \\ ${ }^{3}$ Osaka Medical College, Takatsuki, Japan; ${ }^{4}$ Department of Nursing, Nagoya University of Arts and Sciences, Nagoya, Japan
}

\begin{abstract}
Given the possible medical complications, risks, and the need for informed decision making, pregnancy after kidney transplantation is a serious and difficult issue. In this article, we summarize aspects related to pregnancy counselling for kidney transplant recipients based on past research.

Kidney transplant recipients tend to have overall higher rates of preterm deliveries, low-birthweight babies, and caesarean section than the general population. There have been instances of maternal complications such as preeclampsia, allograft loss, and urinary tract infections during pregnancy; in particular, hypertension is a common problem among kidney transplant recipients.

Pregnancy counselling should start when a recipient chooses renal replacement therapy, and the counselling program should be tailored to the recipient's life cycle and treatment period. It is necessary to explain the essential conditions for pregnancy, such as the need to change to a safe drug, ensure stable kidney function, and wait one to two years after transplantation before conception. Medical staff should be informed about menstrual recovery after transplantation, contraception, and unexpected pregnancy. When a recipient becomes pregnant, it is necessary to convey information about the mode of delivery and breastfeeding.

The contents of pregnancy counselling must be modified according to recipient needs, and detailed discussions are needed between medical staff and transplant recipients. Medical staff should support recipients and their family during the decision-making process before conception. It is essential for kidney transplant recipients to discuss the topic of pregnancy with transplantation physicians, so as to give birth safely and maintain their health with a donated kidney.
\end{abstract}

Keywords: Kidney transplantation; Pregnancy; Nursing; Counseling

\section{INTRODUCTION}

Women with end stage renal disease often have difficulty conceiving through spontaneous pregnancy, and many suffer from infertility [1]. Kidney transplantation has been shown to improve fertility and increase the possibility of pregnancy [1,2]. Therefore, kidney transplantation has become an option for female patients of childbearing age who wish to become pregnant [3]. Recently, the frequency of pregnancies among kidney transplant patients has increased owing to significant improvements in their medical management [4]. However, given the possible medical complications, risks, and the need for informed decision making, pregnancy after kidney transplantation is a serious and difficult issue. Thus, pretransplant and pre-pregnancy counseling for women of childbearing age are increasingly recommended $[1,3,5,6]$. It is important for counseling to reflect the information sought and needed by organ recipients. In this article, we summarize aspects related to pregnancy counseling for kidney transplant recipients based on past research.

${ }^{*}$ Correspondence to: Yuki Yoshikawa, Faculty of Nursing, Shitennoji University, 3-2-1 Gakuenmae, Habikino, Osaka, 583-8501, Japan, Tel: +81(0)72-956-3181, Fax: +81(0)72-956-6011; Email: zippy103yukky@gmail.com

Received: January 31, 2020; Accepted: February 17, 2020; Published: February 24, 2020

Citation: Yoshikawa Y, Uchida J, Kosoku A, Akazawa C, Suganum N (2020) Pregnancy Counseling for Kidney Transplant Recipients. Clinics Mother Child Health. 17:341. DOI: 10.35248/2090-7214.20.17.341.

Copyright: ( $) 2020$ Yoshikawa Y, et al. This is an open-access article distributed under the terms of the Creative Commons Attribution License, which permits unrestricted use, distribution, and reproduction in any medium, provided the original author and source are credited. 


\section{PREGNANCY OUTCOMES IN KIDNEY TRANSPLANTATION}

A recent meta-analysis has shown that the average gestational age was 35.6 weeks and birth weight was 2420 grams [7]. Kidney transplant recipients tend to have overall higher rates of preterm deliveries, low birth weight babies, and caesarean section than the general population $[1,7,8,9,10]$. There have been maternal complications such as preeclampsia, allograft loss, and urinary tract infections during pregnancy; particularly, hypertension is a common problem among kidney transplant recipients [1,7-11]. Hypertension and preeclampsia are the main causes of preterm babies in kidney transplant recipients [9]. Transplantation physicians aim to achieve lower blood pressure for recipients compared with the general population; however, this may affect foetal growth and outcomes in pregnancy. According to a recent report, hypertension during pregnancy had led to small-for-date babies [10]. Hypertension is a problem not only during pregnancy but also pre-pregnancy for kidney transplant recipients. A drug-treated history of pre-pregnancy hypertension is associated with graft loss [9]. Thus, prudent drug treatment by transplantation physicians is important to prevent perinatal hypertension.

Calcineurin inhibitors, namely azathioprine and prednisone, are generally considered safe for use during pregnancy for kidney transplant recipients. Babies of transplant recipients face intrauterine exposure to immunosuppressive agents. Nevertheless, the rate of congenital infections in babies of transplant recipients is not generally higher than in those born to nontransplant recipients. However, primary cytomegalovirus infections during pregnancy have led to a symptomatic congenital cytomegalovirus infection in newborns [9]. Besides, long-term administration of immunosuppressants reportedly pose the risk of attention deficit hyperactivity disorder among children of kidney transplant recipients $[3,11]$. Thus, it is especially important to explain the influence of medicines on the body and foetus to transplant recipients.

\section{PREGNANCY COUNSELLING}

Hypothalamic-pituitary suppression causes amenorrhea in women on dialysis; as a result, they have difficulty conceiving through spontaneous pregnancy, and many suffer from infertility [1,12]. In contrast, kidney transplantation has been shown to improve fertility and increase the possibility of pregnancy [1,2]. Typically, fertility is restored within a few months to one year post-operation [1,7]. Kidney transplant recipients are counselled to wait from one to two years between transplantation and pregnancy, to alleviate the risk of allograft failure [5,9]. Additionally, kidney transplant recipients have to avoid the use of mycophenolic acid or mTOR inhibitors during pregnancy [9]. It is of importance for kidney transplant recipients to discuss the prospect of pregnancy with transplantation physicians, so as to give birth safely and maintain their health with a donated kidney. Furthermore, it is necessary to avoid unwanted pregnancy.

It is important for counselling to reflect the information that organ recipients wish to know and transplantation physicians want to convey. Most recipients seek information on the effects of drugs on the foetus [8], while medical staff recognizes that information on pregnancy risks and timing is of utmost importance [1,3]. In a study by Yoshikawa [8], despite having received pregnancy information from medical staff before transplantation, recipients sought more detailed information. This showed that medical staff's provision of information was not satisfactory. Therefore, the recommendations for pregnancy counselling content after kidney transplantation are shown in Figure 1.

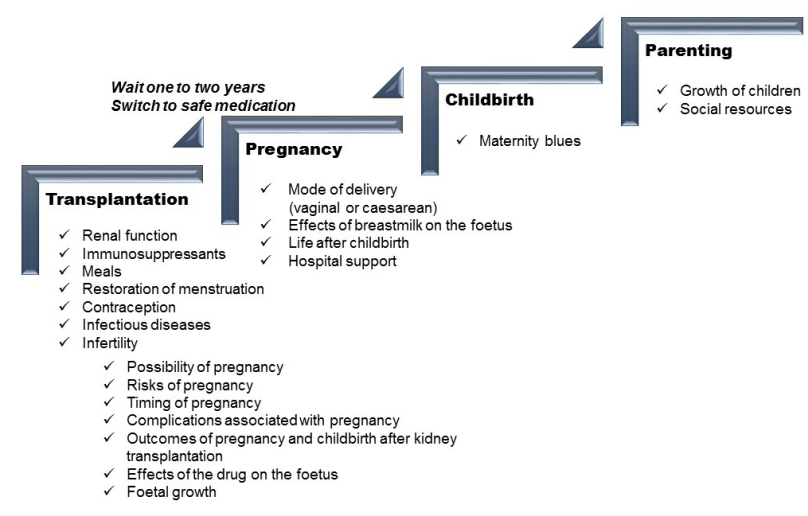

Figure 1: Recommendations for pregnancy counselling content after kidney transplantation

Pregnancy counselling should begin when a patient chooses renal replacement therapy. Although many recipients believe that they can become pregnant with a transplant, there is a possibility of infertility after transplantation. These facts need to be communicated before the transplant, so that patients have realistic expectations from the transplantation procedure. Pregnancy can cause kidney function to deteriorate, and poor renal function before pregnancy leads to allograft loss during pregnancy. It has been reported that creatinine is significantly elevated after pregnancy [13]. Pregnancy should be attempted after renal function has stabilized after transplantation. It is important for the family to plan and discuss the topic of pregnancy before the transplant, which also helps to begin infertility treatment at an early stage. In addition, menstrual recovery after transplantation and contraception methods should be explained to recipients to prevent unexpected pregnancy, previous studies have reported that contraceptive methods for women with renal disease include barrier methods, hormonal methods, and intrauterine devices, but there is limited data on efficacy and failure rates. The choice of contraceptive must be made according to the individual physical condition of the recipient, who should be involved in the decision making.

It is necessary to explain the essential conditions for pregnancy, such as the need to change to a safe drug, ensure stable kidney function, and wait one to two years before conception after transplantation. Although many recipients are concerned about the effects of drugs, there should be sufficient explanation to avoid teratogenic drugs. Research has indicated that mycophenolic acid and calcineurin inhibitor agents pass through the placenta and can be found in foetal circulation [9]. 
Therefore, kidney transplant recipients are recommended to switch from mycophenolic acid to azathioprine six weeks before attempting conception or as soon as possible after an unplanned pregnancy has been confirmed [3,9].

Breastfeeding is also an important aspect to be addressed. Yoshikawa reports that $92.8 \%$ of kidney transplant recipients choose artificial milk [8]. The National Transplantation Pregnancy Registry has reported no specific problems related to breastfeeding in the children of recipients [11]. Immunosuppressant exposure via breast milk is also reported as likely minimal [14]. The benefits of breastfeeding may outweigh the theoretical risks of adverse effects on infants [14]. Past research has reported that many recipients sought detailed information on breastfeeding [8], and they may have selected artificial milk because of the absence of concrete explanation. Medical staff should explain the advantages and disadvantages of breastfeeding and breastfeeding options before delivery.

Urinary tract infection is the most frequently encountered infection in pregnant kidney transplant recipients. It is caused by complicated factors, which may lead to preterm delivery. Although vaginal delivery is not contraindicated as a delivery method, caesarean section is selected in case of obstetric adaptation [1]. The medical staff should explain the high possibility of preterm delivery due to obstetric complications, as well as the risks of a caesarean section. The midwife should likewise offer counselling regarding preventing a preterm delivery, including prevention of infection and self-management.

Considering that a lot of information needs to be provided to transplant recipients, timely explanation is important. The recipient is often in a situation where the medical staff is too busy to be asked questions. In Japan, there is a tendency to avoid talking about sexual life owing to the cultural background, and many recipients tend to have insufficient discussions. Cultural backgrounds vary from country to country; however, the healthcare providers' approach is important. It is also necessary to provide information not only to the kidney transplant recipient but also to his/her partner to ensure the recipient has support and companionship during the counselling process. Support from the medical staff and the family are equally important for a safe perinatal period.

\section{CONCLUSION}

It is necessary to provide pregnancy counselling according to the recipient's life cycle and treatment period. In addition, the contents of pregnancy counselling must be modified according to recipient needs, and detailed discussions are needed between medical staff and transplant recipients. Medical staff should support the decision making of recipients and their family regarding conception.

\section{FUNDING}

This study was supported by the Japan Society for the Promotion of Science, Grants-in-Aid for Scientific Research Number JP25870952.

\section{REFERENCES}

1. Fuchs KM, Wu D, Ebcioglu Z. Pregnancy in renal transplant recipients. Seminars in Perinatology. 2007;31(6):339-347.

2. Armenti VT. Pregnancy after transplantation: milestones and assessments of risk. Am J Transplant. 2011;11(11):2275-2276.

3. Concepcion BP, Schaefer HM. Caring for the pregnant kidney transplant recipient. Clin Transplant. 2011;25(6):821-829.

4. McKay DB, Josephson MA. Pregnancy in recipients of solid organs: effects on mother and child. N Engl J Med. 2006;354(12): 1281-1293.

5. Gill JS, Zalunardo N, Rose C, Tonelli M. The pregnancy rate and live birth rate in kidney transplant recipients. Am J Transplant. 2009;9(7):1541-1549.

6. Tong A, Brown MA, Winkelmayer WC, Craig JC, Jesudason S. Perspectives on pregnancy in women with CKD: A semistructured interview study. Am J Kidney Dis. 2015;66(6):951-961.

7. Deshpande NA, James NT, Kucirka LM, Boyarsky BJ, GaronzikWang JM, Montgomery RA, et al. Pregnancy outcomes in kidney transplant recipients: A systematic review and meta-analysis. Am J Transplant. 2011;11(11):2388-2404.

8. Yuki Y, Junji U, Chiharu A, Nobuhiko S. Outcomes of and perspectives on pregnancy counseling among kidney transplant recipients. Transplantation Reports. 2019;4(1):1-5.

9. Chittka D, Hutchinson JA. Pregnancy after renal transplantation. Transplantation. 2017;101(4):675-678.

10. Blume C, Sensoy A, Gross MM, Guenter HH, Haller H, Hermann $\mathrm{H}$, et al. A comparison of the outcome of pregnancies after liver and kidney transplantation. Transplantation. 2013;95(1):222-227.

11. Coscia LA, Constantinescu S, Moritz MJ, Frank AM, Ramirez CB, Maley WR, et al. Report from the national transplantation pregnancy registry (NTPR): outcomes of pregnancy after transplantation. Clin Transpl. 2010:65-85.

12. Mitani M, Matsuda Y. Reproductive endocrinology and pregnancy in renal transplant recipients. Sanfujinka Chiryo. 2011;102:717-724.

13. Shah S, Venkatesan RL, Gupta A, Sanghavi MK, Welge J, Johansen R, et al. Pregnancy outcomes in women with kidney transplant: Metaanalysis and systematic review. BMC Nephrol. 2019;20(1):1213-1215.

14. Fitzpatrick A, Mohammadi F, Jesudason S. Managing pregnancy in chronic kidney disease: Improving outcomes for mother and baby. Int J Womens Health. 2016;8:273-285. 